\title{
Non-neoplastic glandular structures in bone marrow: a technical artefact
}

\author{
W G McCluggage, R Clarke, H Bharucha
}

\begin{abstract}
A case of non-neoplastic glandular structures embedded in the centre of a bone marrow trephine biopsy is described. This is a hitherto unreported phenomenon. These glandular structures originated from the dermal sweat gland and represented an artefactual lesion produced by the biopsy procedure. The importance of considering the possibility of non-neoplastic glandular structures in bone marrow is stressed, in order to avoid a misdiagnosis of metastatic adenocarcinoma.
\end{abstract}

(f Clin Pathol 1995;48:1141-1142)

Department of

Hospitals Trust, Belfast

W G McCluggage

Keywords: Bone marrow, non-neoplastic glandular structures.

\section{Department of}

Pathology, Craigavon Area Hospital,

Craigavon

R Clarke

Department of

Pathology,

The Queen's

University of Belfast

$\mathrm{H}$ Bharucha

Correspondence to: Dr W G McCluggage Department of Pathology, Royal Group of Hospitals Trust, Grosvenor Road, Belfast BT12 6BL.

Accepted for publication 1 June 1995
Non-neoplastic glandular epithelial structures are well known and have been described in lymph nodes. These inclusions are presumed to represent glandular structures which have been entrapped during embryological development. $^{1}$ Non-neoplastic glandular inclusions have been reported in pelvic ${ }^{1}$ and axillary lymph nodes ${ }^{2}$ as well as in nodes around thyroid ${ }^{3}$ and salivary glands. ${ }^{4}$ The differential diagnosis and distinction from metastatic carcinoma has been stressed. As far as we are aware, non-neoplastic glandular structures have not previously been described in bone marrow.

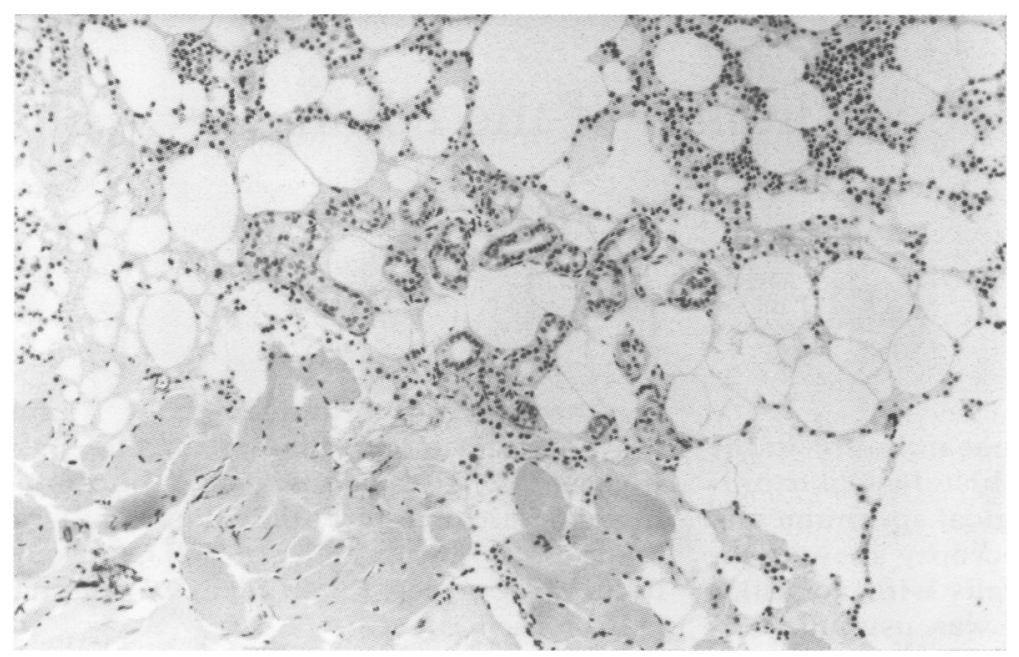

Figure 1 Non-neoplastic glandular structures and adjacent skeletal muscle embedded in adipose tissue and haemopoietic cellular elements.

\section{Case report}

A 64 year old woman underwent a bone marrow trephine biopsy during investigation for longstanding hypochromic, microcytic anaemia. A biopsy specimen was taken from the posterior iliac crest using a Jamshedi second generation needle.

Histology of the trephine biopsy specimen showed a mild hyperplasia of erythroid cells in keeping with the history of anaemia. In the centre of the bone marrow several glandular structures, surrounded by adipose tissue and haemopoietic cells, were identified (fig 1). These were composed of cuboidal cells surrounding central lumina. Nuclei were bland with no evidence of pleomorphism or mitotic activity. On high power examination, a second attenuated layer of myoepithelial cells was seen surrounding the cuboidal cells in several of the glandular structures (fig 2). There was no associated stromal desmoplasia. Close to the glandular structures, again within the centre of the marrow, several fragments of skeletal muscle, with identifiable cross striations, were present.

Immunohistochemical staining showed the cells lining glandular lumina to be strongly positive with Cam 5.2 and antibodies directed against epithelial membrane antigen. The myoepithelial cell layer showed positive staining with Cam 5.2 and $\alpha$-smooth muscle actin.

\section{Discussion}

Non-neoplastic glandular epithelial inclusions have been described in several lymph node groups, chiefly pelvic and peri-aortic, ${ }^{1}$ and axillary nodes ${ }^{2}$ in women. Non-neoplastic salivary ${ }^{3}$ and thyroid ${ }^{4}$ epithelial inclusions may also be found in lymph nodes in the vicinity of these organs in both sexes. The origin of these inclusions has been debated. Pelvic and periaortic inclusions are thought to arise from metaplastic proliferation of peritoneal mesothelium which becomes entrapped in nodal structures during embryological development. ${ }^{1}$ Similarly, salivary and breast inclusions within axillary nodes are thought to arise following entrapment during embryological development. ${ }^{2}$ Such inclusions are usually found in the subcapsular or cortical areas of nodes, a site where metastatic carcinoma is frequently found. The importance of avoiding a misdiagnosis of metastatic adenocarcinoma has been stressed. Problems may also arise in misinterpreting well differentiated metastatic carcinoma as non-neoplastic glandular inclusions. This is especially true in the case of follicular thyroid inclusions, most of which 


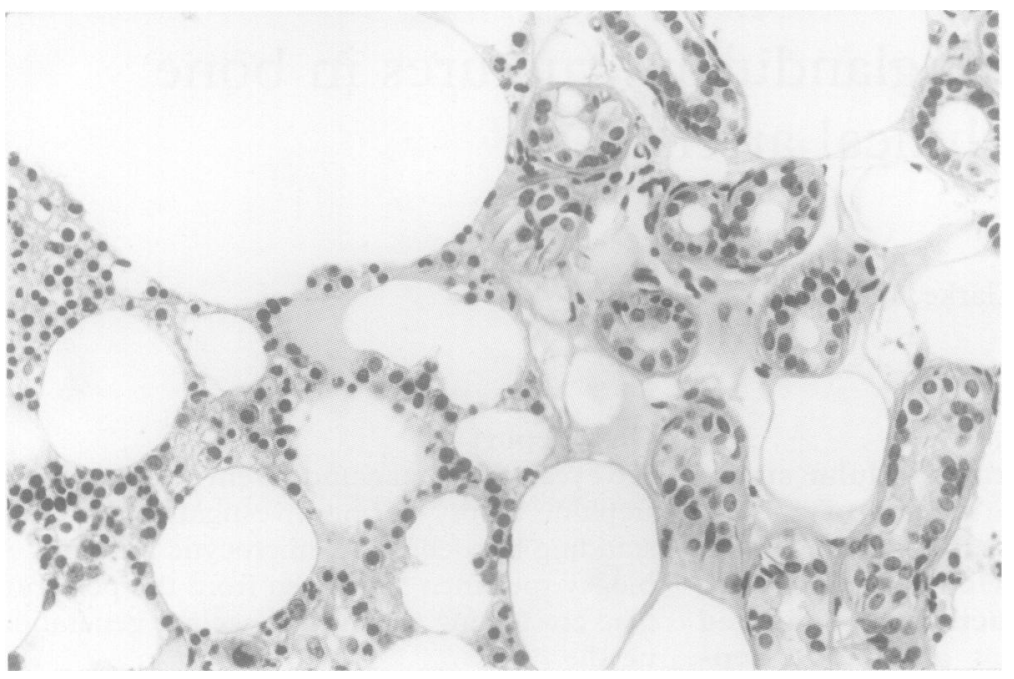

Figure 2 High power showing glandular structures composed of a double layer of epithelial and myoepithelial cells.

represent metastasis from an unsuspected and undiscovered occult thyroid carcinoma.

The presence of glandular elements embedded in the centre of the bone marrow in the present case initially lead to consideration of a diagnosis of metastatic adenocarcinoma. However, metastatic tumour was ruled out due to the lack of the nuclear features of malignancy and of a stromal desmoplastic response and the presence of a double layer of epithelial and myoepithelial cells in several of the glandular structures. Clinical examination and limited radiological investigation did not reveal a primary site of malignancy. The epithelial nature of the structures was confirmed on immunohistochemistry and positive staining for $\alpha$ smooth muscle actin highlighted the presence of a layer of myoepithelial cells.

We believe these non-neoplastic glandular structures to be an artefact produced by the trauma of the biopsy procedure. Small fragments of squamous epithelium derived from skin are a well described artefact in bone mar- row trephine biopsy specimens, especially when performed by an inexperienced operator. Such fragments are generally found at the end of a trephine biopsy specimen and their occurrence has been described in many of the textbooks of bone marrow pathology. ${ }^{5}$ However, the presence of non-neoplastic glandular structures in bone marrow has not been stressed. A further unusual point in the present case was that glandular structures were embedded in the centre of the bone marrow, completely surrounded by haemopoietic elements. Morphologically, they resembled dermal sweat gland structures. The presence of adjacent fragments of skeletal muscle, also embedded within the centre of the marrow, led us to conclude that both glandular and muscular elements had been displaced there during the biopsy procedure. Although skeletal muscle fibres were present in this instance, it is possible that cases might be encountered where glandular structures are present without muscle elements. If a serious misdiagnosis of metastatic malignancy and unnecessary investigation is to be avoided, pathologists and haematologists should consider non-neoplastic glandular structures in cases where epithelial elements show bland nuclear features and an absence of stromal desmoplasia. Such epithelial elements are likely to be of dermal sweat gland origin.

We would like to thank Dr S Nelson (Consultant Haematologist, Craigavon Area Hospital) for his assistance in providing clinical details for this patient.

1 Shen SC, Bansal M, Purrazzella R, Malviya V, Strauss L. Benign glandular inclusions in lymph nodes, endosalpingiosis and salpingitis isthmica nodosa in a young girl with clear cell adenocarcinoma of the cervix. Am $\mathcal{f}$ Surg with clear cell adenoca

2 Holdsworth PJ, Hopkinson JM, Leveson SH. Brief report: benign axillary epithelial lymph node inclusions - a histological pitfall. Histopathology 1988;13:226-8.

3 Goodman MD, Daly JF, Valenis Q. Heterotopic salivary tissue and branchial cleft sinus. Laryngoscope 1981;91:260 4.

4 Meyer JS, Sternberg LS. Microscopically benign thyroid follicles in cervical lymph nodes. Serial section study of lymph node inclusions and entire thyroid gland in five cases. Cancer 1969;24:302-11

5 Frisch B, Lewis SM, Burkhardt R, Bartl R. Introduction (Chapter 1). In: Biopsy pathology of bone and bone marrow. London: Chapman and Hall, 1985:13.

\section{Department of Pathology, Karolinska Institute, 17177 Stockholm, Sweden C A Rubio \\ Correspondence to: Dr C A Rubio. \\ Accepted for publication 1 June 1995}

\begin{abstract}
A case of clear cell adenocarcinoma of the colon is reported. The histological examination of both the surgical specimen and of the metastases at necropsy showed columnar or polygonal cells with vesicular nuclei. The cytoplasm was usually clear with multiple, often empty looking vacuoles. From a panel of histochemical and immunohistochemical reactions,
\end{abstract}

carcinoembryonic antigen and tissue polypeptide antigen showed strong positivity. The histochemical and immunohistochemical differential diagnosis with another common clear cell tumour, namely clear cell renal adenocarcinoma, is discussed.

(f Clin Pathol 1995;48:1142-1144)

Keywords: Clear cells, adenocarcinoma, colon. 